\section{Quantification of Virus Suspensions by Direct Particle Counting}

\author{
Paul R. Hazelton \\ University of Manitoba, Winnipeg, Manitoba, Canada \\ paul_hazelton@umanitoba.ca
}

In science, there are occasions when it is of value to know the number and distribution of different types of particles present in a solution. Calculation of these values is simplified if you can uniformly distribute the number of particles in a known volume over an established surface area. A number of procedures have been proposed. The best which we have used is direct centrifugation of particles to formvar/carbon-formvar coated electron microscope grids in an EM-90 rotor for the Beckman Airfuge ${ }^{\circ}$. This instrument makes use of the ability to pellet all material in a suspension using established centrifugation theory. Radial design of the side walls of the cells prevents accumulation of material on peripheral surfaces and permits even distribution of all material across the total surface area of the circumferential surface of the cell. In addition to the improved sensitivity we have experienced in viral diagnosis ${ }^{1}$, the knowledge obtained with this procedure over the years has permitted us to confirm the relative infectivity of Reovirus Types 1 (Lang) and 3 (Dearing) as between 9 and 14 particles per plaque forming unit 2,6 , dissect the assembly process of reovirus by establishing the presence and distribution of a previously unknown assembly intermediate in permissive and restrictive growth conditions ${ }^{2}$, determine the relative ratio of particles to RT-PCR units in norovirus positive samples at 1.8 particles per RT-PCR unit, and accurately estimate viral load over time in naturally occurring norovirus infections ${ }^{2 a}$.

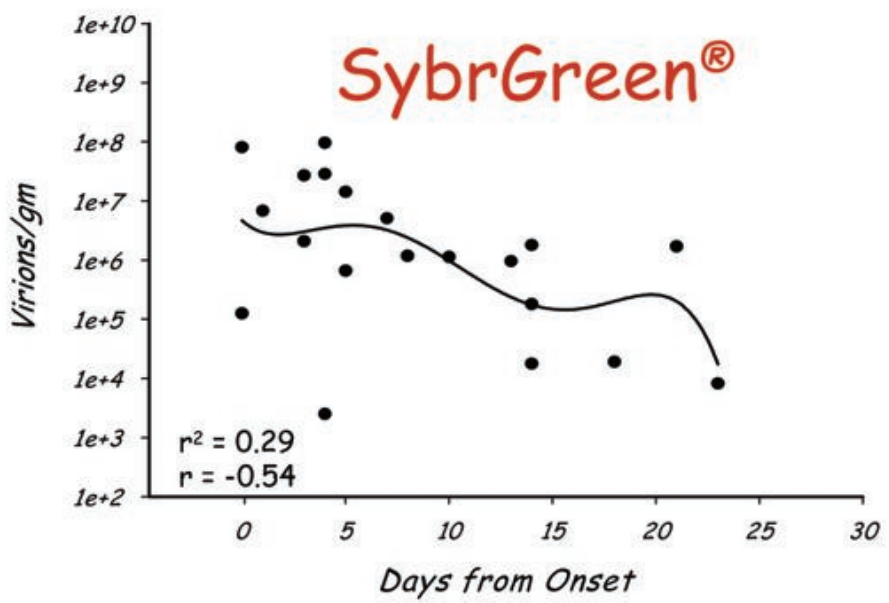

Figure 1. Determination of viral load over time in a population infected with norovirus. To determine the viral load the number of virions/gm was determined for control samples by pelleting particles directly to a formvar coated copper grid with a Beckman Airfuge using an EM-90 rotor, followed by particle counting as described below. The number of PCR units/control sample was determined by qRT-PCR using a Roche LightCycler and the number of PCR units/virion then calculated2a.

Determining the concentration of particles in a solution involves several steps:

1. Count the number of particles in a known surface area;

2. Determine the ratio between the total area and the actual area counted;

3. Multiply the ratio of total area by the number of particles counted to determine the number of particles present in the total area;
4. Divide the total number of particles by the volume of suspension to obtain the concentration of particles $/ \mathrm{mL}$; and

5. Multiply the concentration of particles/mL by the dilution factor of the suspension to obtain the concentration of particles/ $\mathrm{mL}$ in the original sample.

The number of particles per unit area of support can be determined after a uniform deposition of all particles in a known volume over a known surface area. This is done from either thin sections of filters in cross section ${ }^{4}$, or negative stain preparations of grids ${ }^{1,5}$. Counts may be done either directly on the electron microscope viewing screen or from electron micrographs.

The following procedures are adapted from Hammond et al., 1981, and are essentially as described in Hammond et al., 1981, and Hazelton and Coombs, $1999^{1,2}$. Final modifications to the formula originally devised by M.F. Miller are the result of discussions between G. W. Hammond and P. R. Hazelton, Cadham Provincial Laboratories and the University of Manitoba, both in Winnipeg, and C. Lee of Beckman Scientific Instruments, Palo Alto in 1981 .

\section{Sample preparation}

1. For clinical samples prepare a suspension as per:

a. Liquid samples (blood, urine, etc.) require no preliminary preparation;

b. Semi-solid samples (feces, some tissues);

i. Combine 1 part sample with 10-20 parts distilled water in a sterile conical centrifuge tube (volume 1-4 mL),

ii. Add 10-12 sterile glass beads,

iii. Vortex for 1-2 minutes to break up the sample, and

iv. Allow the sample to sit on ice for 5 minutes, then repeat the vortex step.

c. Solid samples;

i. Place 1 part sample in a ground glass homogenizer with 9 parts water,

ii. Homogenize the tissue with 6-10 strokes of the ground glass pestle, and

iii. Transfer the homogenate to a sterile, $15 \mathrm{~mL}$ conical centrifuge tube.

2. For tissue culture material:

a. Infect an appropriate cell line at a pre-determined multiplicity of infection (MOI) and incubate at the appropriate temperatures for the desired time;

b. Remove cells from the incubator and place at $-80^{\circ} \mathrm{C}$;

c. Freeze thaw the cells $3 x$; and

d. Transfer the suspension to a sterile, $15 \mathrm{~mL}$ centrifuge tube.

3. Clear large debris by centrifugation at $800 \mathrm{xg}$ for 5 minutes at $4^{\circ} \mathrm{C}$.

4. Remove an aliquot of suspension for determination of titre of infectious virus as per standard lab procedures.

5. Where virus is free in the supernatant (either clinical sample or tissue culture preparation):

a. pre-clear $0.2-0.5 \mathrm{~mL}$ of the suspension at $15,000 \mathrm{xg}$ for 1-5 minutes in a benchtop microcentrifuge (e.g., Eppendorf model 5412 or 5415 benchtop centrifuge); and

b. Carefully remove as much of the supernatant as possible and transfer it to a sterile, silicone treated tube, leaving the pellet undisturbed. (See $a, b$ in NOTES section)

6. Where the virus may be bound to cellular material do not preclear the viral suspension.

7. Add fixative to the supernatant, vortex well $(\sim 10 \mathrm{sec})$ and allow the virus to stabilize for 10 minutes at $4^{\circ} \mathrm{C}$ : 


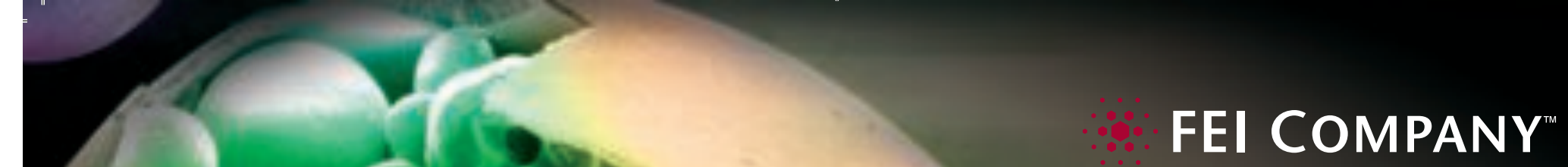

\section{FEI COMPANY}

TOOLS FOR
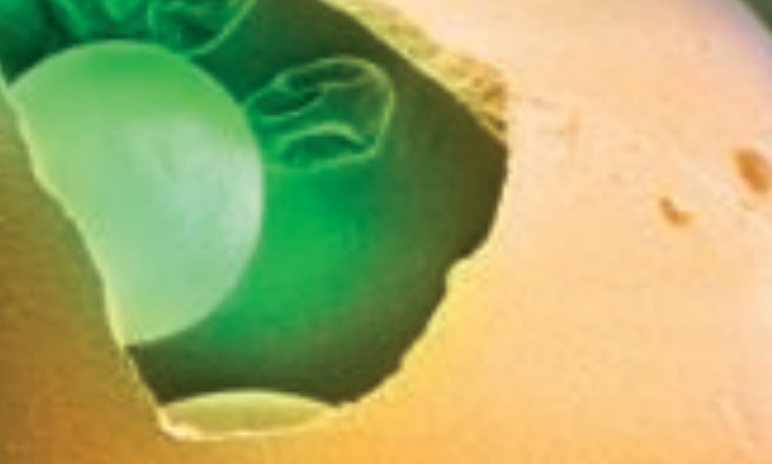

The next-generatior
for nano-pioneers

The Helios NanoLab ${ }^{\text {TM }}$, FEl's next generation DualBeam ${ }^{\text {TM, }}$, is in a class of its own when it comes to imaging, analysis and control of matter on the nanometer level. It produces the thinnest samples of the highest quality in a quick and easy way. It excels in preparing samples for almost any SEM/FIB application, with minimal damage to the sample. It pushes the limits in 2D and 3D nano characterization to deliver the most advanced integrated solutions for nanoprototyping. Its Titan ${ }^{\mathrm{TM}}$-derived platform features unsurpassed deep nanometer resolution SEM performance to provide imaging and contrast of the highest quality with the best stability at the highest speed. All in all, Helios NanoLab is the perfect tool for pioneers who want to expand the boundaries in nanotechnologies.

www.fei.com sales@fei.com

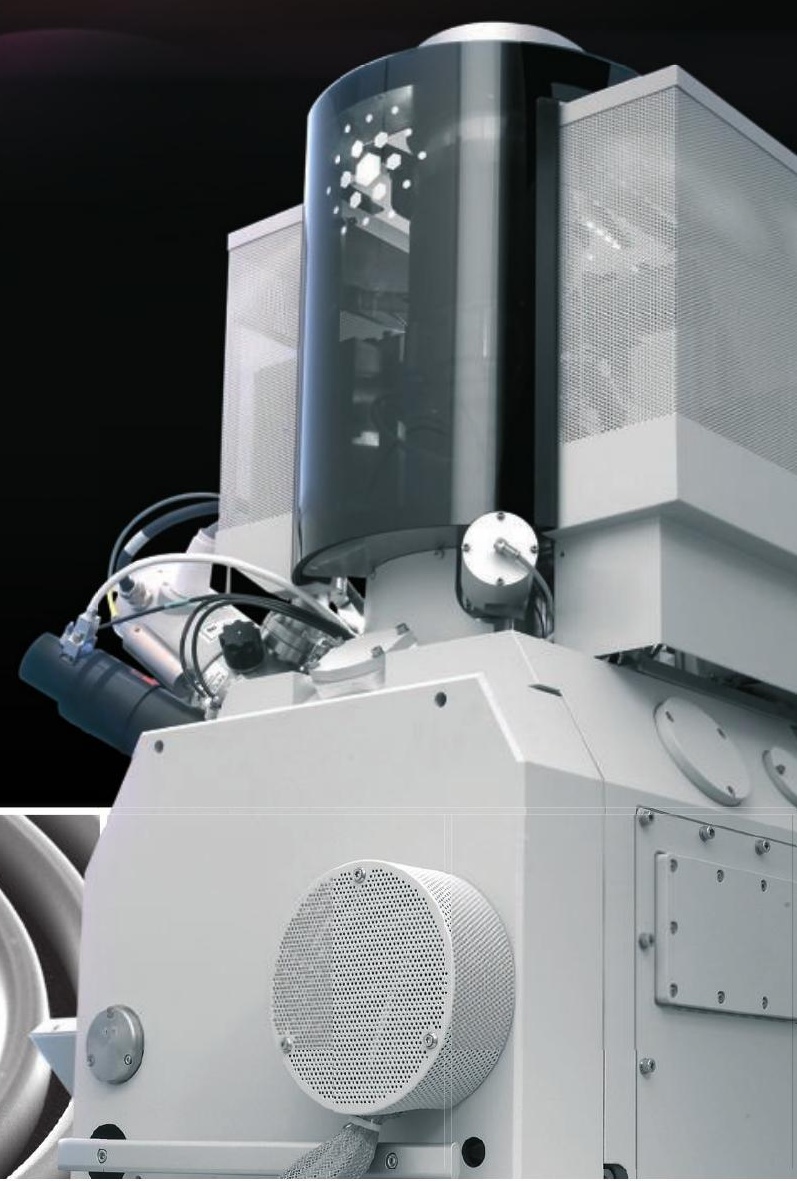


a. Paraformaldehyde to a final concentration of $2 \%$; or

b. Glutaraldehyde to a final concentration of $0.1 \%$. (See $c$, $d$ in NOTES)

8. Where the sample may still contain small, light particulate material, such as membranes and lipids seen with tissue culture fluids, do a final clearance of the suspension as per:

a. Put $50 \mu \mathrm{L}$ precleared suspension on a $100 \mu \mathrm{L} 30 \%$ sodium tartrate cushion ( $\mathrm{pH} 7.2$ ) in A100 airfuge tubes

b. Pellet viral components by airfuging for 1 hour at 26 ppsi in an A100 rotor.

c. Carefully withdraw most of the Tartrate cushion and TCF. Pipette from the top, at meniscus level to ensure that you remove material in the cushion/TCF fraction. To ensure the pellet is not disturbed, leave about $5 \mu \mathrm{L}$ in the tube.

d. Resuspend the pellet in $50 \mu \mathrm{L}$ of the same buffer and fixative used for the initial fixation procedure.

9. Prepare a series of 10 fold dilutions of the sample in the same buffer and fixative used for the initial fixation procedure. Sterile siliconeized tubes should be used.

Centrifugation directly to the electron microscope specimen grid

10. Place a formvar coated 400 mesh grid in each well of a sector core base for the Airfuge EM-90 rotor, formvar surface towards the center of the sector core base. (See $e$ in NOTES)

11. Put $45 \mu \mathrm{L}$ fixed suspension in separate wells for each sample/dilution which is to be enumerated.

12. Lightly grease a sector core gasket with high grade vacuum grease and place the gasket on the base of the sector core base. Put the sector core lid on the gasket to complete the sector core assembly. (See $f$ in NOTES)

13. Put the sector core assembly into the rotor base. Put the rotor lid on the base and tighten the lid. (See $g$ in NOTES)

14. Pellet biomolecules in the suspension directly to the grid for 30 minutes at 26 psi. (See $h, i$ in NOTES)

15. Remove the grid from the sector core taking care to only handle the grid at the edges.

16. Touch a freshly torn edge of Whatman \#1 filter paper to the edge of the grid to remove excess fluid. Take care to also touch the back surface of the grid to remove any fluid which is on that surface. (See $j$ in NOTES)

17. Stain the grid with $2.5 \mathrm{mM}$ Phosphotungstic acid, $\mathrm{pH} 7.0$ as per standard laboratory procedures.

\section{Particle counting}

18. Enumerate viral and subviral components in 5 separate grid squares as per: (See $k$ in NOTES)

a. Arbitrarily divide the grid into quarters;

b. Select the first complete square at the outside edge of each quadrant. If that square is partially obscured or the film is damaged, then check the surrounding squares in an outward spiral until the first suitable square is identified for the quadrant;

c. For the fifth square, find the center marks for the grid and check the surrounding squares in an outward spiral until the first suitable complete center square is identified;

d. Determine the approximate quantity of particles in each square using a $0-4+$ scale where;

i. $0=0$ particles/square,

ii. $1+=1-10$ particles/square,

iii. $2+=10-100$ particles/square,

iv. $3+=100-1000$ particles/square, and

v. $4+>1000$ particles/square.

e. Directly count all particle, subcomponent structures and other structures of interest in each square directly on the electron microscope viewing screen in each of 5 grid squares for the dilution determined to contain between 20 and 200 particles per square. (See $\ell$ in NOTES)

19. Determine the average number of particles and subviral components per grid square.

\section{Calculating the concentration of particles $/ \mathbf{m L}$}

20. Determine the total number of particles/mL using the following equation:

$$
N=V_{f} \frac{D}{V o l} \times \frac{B}{A}
$$

Where:

$N=$ total number of particles $/ \mathrm{mL}$;

$V_{f}=$ number of particles/grid square;

Vol = volume tested in $\mathrm{mL}$;

$D=$ dilution factor (as the inverse. Therefore, with a 1:200 dilution $D$ would be 200 .);

$B=9 \mathrm{~mm}^{2}$, or $9 \times 10^{6} \mu \mathrm{m}^{2}$ for the $50 \mu \mathrm{L}$ well sector core, and

$A=$ average area counted

The average area of the grid squares for Gilder 400 mesh grids is $1.12 \times 10^{3} \mu \mathrm{m}^{2}$. Where these grids are used and the standard volume of $45 \mu \mathrm{L}$ is placed in each well for the small well sector core base (total volume $=50 \mu \mathrm{L}$ ), then the formula can be shortened as per:

$$
\begin{gathered}
N=V_{f} \frac{D}{0.045} \times \frac{9 \times 10^{6}}{1.12 \times 10^{3}} \\
N=V_{f} \times D \times 1.79 \times 10^{5}
\end{gathered}
$$

Or, essentially, 1 particle/square $=1.79 \times 10^{5}$ particles $/ \mathrm{mL}$ in the suspension that was centrifuged.

\section{Leakage in the sector core}

\section{If there is leakage}

a. Check that the gasket has been properly greased.

b. If leakage continues replace the gasket with a new one

c. If leakage still continues, and the same wells are always the source of leakage, there is probably a minute crack in one or more wells of the sector core. Replace the sector core with a new one.

\section{NOTES:}

a. All viruses will be cleared from suspension in a centrifugal field if they are centrifuged long enough. Therefore, the centrifugation time for pre-clearing large material from a suspension while small material remains in solution is a function of the degree of clumping and the sedimentation coefficient of the virus involved. During the initial investigations it was determined that preclearing at $15,000 \mathrm{rpm}$ for 
The State of the Art, Revolutionary

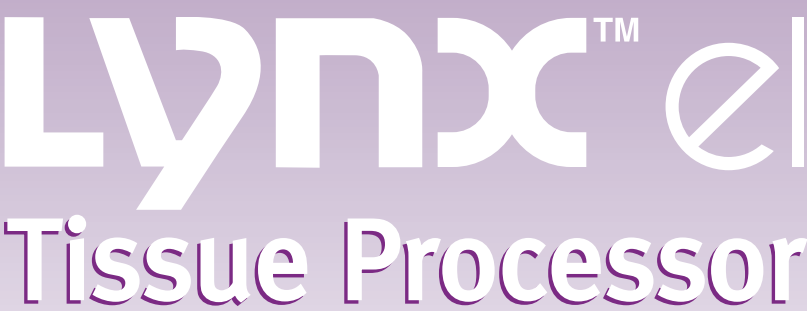

The EMS LYNX Automated Tissue Processor now made even better... for Electron Microscopy and Light Microscopy.

Dramatically reduce your sample preparation cost and time with improved reproducible results!!

The EMS LYNX allows for the processing of tissue all the way through $100 \%$ resin.

\section{Primary uses:}

- Ultrastructural Studies

- Extremely Hard Tissues - Undecalcified Bone

- High Resolution Light Microscopy

- Immunohistochemistry

- Dehydration for SEM

- Deparaffinization

- En-bloc Staining

- And Much More

This state of the art Automated Tissue Processor has many unique features making the unit the most versatile and reliable unit on the market at a very competitive price.

\section{Features:}

- Stainless Steel Working Surfaces

- Enclosed Benchtop Unit

- Reagent Turntable/ Max of 56 Specimens

- 20 Reagent Vials/Cycle

- Temp Range of $4^{\circ} \mathrm{C}-60^{\circ} \mathrm{C}$

- Built in Exhaust Fan

- Alarm Monitoring System

- Multiple Safety Features

- Battery Back Up

- Storage of 10 Programs/

Each with 20 Pre-programmable Steps

- Printer Output

- Delay Start of Programs

- And Much More...

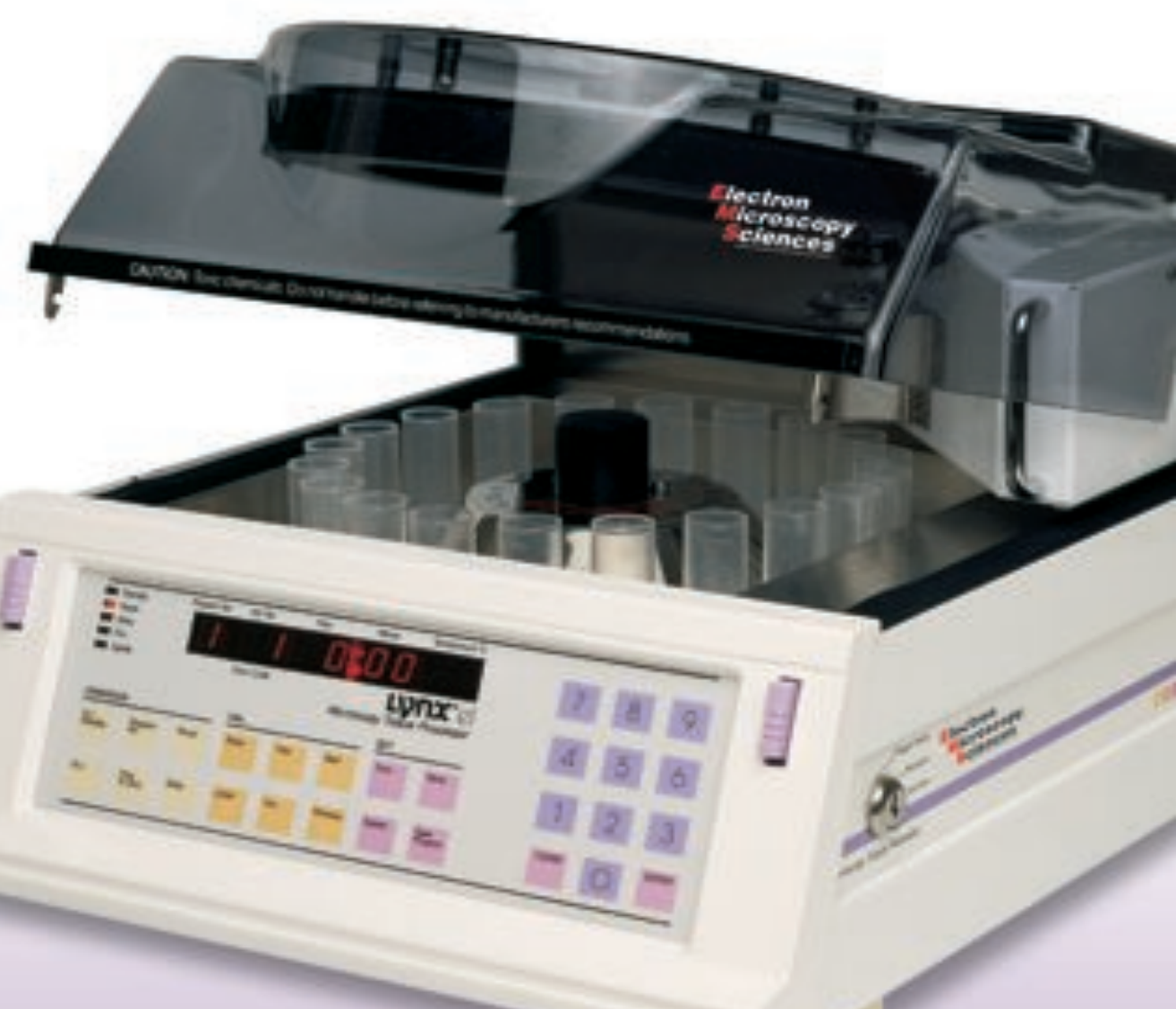

For more information or a complete brochure on the "Lrar" Automated Tissue Processor, please call or write us today. 
1-5 minutes in a standard benchtop microcentrifuge (e.g. Eppendorf 5412) did not reduce the particle yield, while preclearing for 15 minutes depleted the level of rotavirus single virions and clumps in suspension.

b. Silicone treated tubes should always be used to limit the possibility that material will adhere to the side of the tube and be removed from suspension

c. Early studies in this lab showed that the outer capsid shell of unstabilized rotavirus is removed from the virions by shear forces created within structures in centrifugal fields. Stabilization with $0.1 \%$ final concentration glutaraldehyde or $2 \%$ final concentration paraformaldehyde is sufficient to prevent damage to virions. Treatment with glutaraldehyde at a concentration of $0.1 \%$ will also leave antigenic sites in their native, antigenic state, thereby allowing immuno elec-

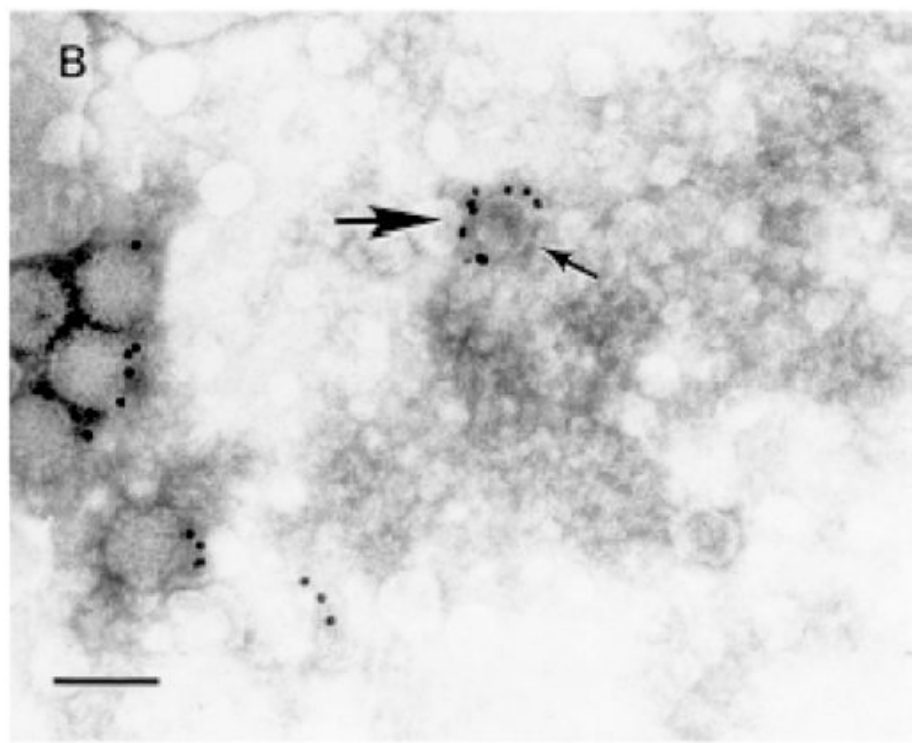

Figure 2. Direct immunoelectron microscopy of subviral components. L929 cells were infected with Reovirus mutant tsA279, freeze-thawed, cleared through tartrate, stabilized with $0.1 \%$ glutaraldehyde, and pelleted directly to a formvar coated nickel grid as described in the procedures. Gold beads were prepared, antiserum conjugated to the beads, and direct immunoelectron microscopy conducted as described elsewhere2.

tron microscope procedures to be conducted, ${ }^{2,7}$ figure 2 .

d. Stabilization of viral structures with tissue fixatives such as glutaraldehyde and formaldehyde is due to formation of chemical bonds that cross link different areas within a molecule, or between different molecules in the multimeric biomolecule. Dogma states that because glutaraldehyde is a 5 carbon chain with highly reactive carboxyl groups at each end it is more likely to cross link different virions, or virions with cellular detritis, than formaldehyde, which has a single carbon and a single reactive aldehyde group. While we have not observed any appreciable clumping using glutaraldehyde at a concentration of $0.1 \%$, the possibility of artificial clumping must not be ignored. Some labs quench the crosslinking activity of glutaraldehyde after a short fixation period by the addition of glycine. Because lysine can contribute to crosslinking it should not be used as a substitute. The final concentration of glycine in the preparation should be $8 \mathrm{mM}$ for $0.1 \%$ glutaraldehyde and $125 \mathrm{mM}$ for $2 \%$ paraformaldehyde suspensions. Freshly prepared paraformaldehyde or quality controlled commercial preparations of EM grade paraformaldehyde is preferable to purchased formaldehyde solutions. We have not confirmed retention of antigenicity after paraformaldehyde stabilization.

e. Why 400 mesh grids? Because the size of the grid square is small enough to allow accurate photographic measurement of the total open area in the square at low magnification. Lower mesh grids have openings which are too large for accurate photographic measurement of the openings, while the openings in higher mesh grids reduce the effective area counted affecting reliability and accuracy of quantification.

f. The amount of grease used on the gasket must be kept to a minimum. Otherwise grease may also be 'pelleted' to the grid by the centrifugal forces. It should be possible to faintly see a fingerprint in the grease on the gasket.

g. It is impossible to overemphasize the need for care to prevent cross contamination of wells during this whole procedure. Do not try to assemble the sector core assembly inside the rotor base. It is virtually impossible to avoid cross-well contamination if you try that. Too much grease on the gasket will also contribute to the possibility of cross contamination

h. The Beckman Airfuge is rated to run at a maximum air pressure of 30 psi. During the original study the air pressure for the building provided delivery of a maximum air pressure of 26 psi. To determine the $K$ factor for the reduced speed the following formula is used:

$$
K_{a c t}=K_{\max } \frac{r p m_{\max }}{r p m_{a c t}}
$$

Where:

$K_{\max }=$ the $K$ factor at the maximum speed. For the EM-90 Rotor $K_{\text {max }}=19$;

$K_{a c t}=$ the $K$ factor at the actual speed; $r p m_{\text {max }}=$ rpm at maximum speed (in 1,000's).

$R p m_{\text {max }}=93$ (x1,000); and

$R p m_{a c t}=\mathrm{rpm}$ at actual speed (in 1,000's). At $26 \mathrm{psi}$, $r p m_{a c t}=83(\mathrm{x} 1,000)$.

$$
K_{\text {act }}=19 \times \frac{93}{83}=21.3
$$

i. In the original study we used the size of the hepatitis B $45 S_{20 w}$ particle to calibrate the clearing time to pellet all particles to the grid. To date, no smaller virion particle has been identified. However. If a smaller particle must be used then the following equations will help define the amount of time needed for pelleting. To determine the time needed:

$$
T=\frac{K_{a c t}}{S_{20 w}}
$$

Where:

$T=$ time (in hours);

$K_{a c t}=K$ factor for the rotor at the speed used; and

$S_{20 w}=$ Sedimentation coefficient for the molecule of interest. For the hepatitis B $45 S_{20 W}$ particle the calculations are: 


$$
T=\frac{21.3}{45}=0.47 h=28.4 \mathrm{~min}
$$

j. It is essential that the back of the grid be dried by wiping with the fresh torn edge of filter paper. The grid will be wet on both surfaces when it is removed from the sector core well. If the grid is put directly on the drop of stain while the back surface is wet the grid may immerse in the stain. At a minimum the liquid on the back surface will merge with the stain and allow it to wet the back surface. When excess stain is wicked away stain will pool in the area between the grid bar and the plastic film. There are two problems that arise. First, the accumulation of stain creates an electron dense area. When electrons cannot pass through this dense area, energy is transferred to the stain, energy becomes heat energy, the plastic film gets hot, and breaks. Even if the film does not break, the open area of the grid is reduced, giving a characteristic rounded appearance to the square. Because the area is reduced, you cannot accurately determine the number of particles in an entire square.

k. Five well dispersed grid squares are counted to guarantee that local grid environmental factors are balanced. For example, large clumps may be enriched to one side of the grid due to inertial effects during start of operation, or there may be localized damage to the formvar-carbon film in the handling of the edge of the grid.

1. The reason for picking a grid square with 20-200 particles is to provide a large enough population to provide reproducibility ( $\geq 20$ particles) but with few enough to prevent double counting particles during the process of scanning across the total area of the grid square.

\section{References:}

1. Hammond, G.W., Hazelton, P.R., Chuang, I., and Klisko, B. (1981). Improved detection of viruses by electron microscopy after direct ultracentrifuge preparation of specimens. J. Clin. Micro. 14:210, 221.

2a. P.R.Hazelton, P.R. Ball, T.B. and Hammond, G.W. SybrGreen ${ }^{7}$ and Taq$\mathrm{Man}^{7}$ probe real-time PCR to determine calicivirus shedding duration and viral load. Second World Calicivirus Conference, Dijon, France, 6-9 November, 2004.

2. Hazelton, P.R. and Coombs, K.M. (1999). The reovirus mutant tsA279 L2 gene is associated with generation of a spikeless core particle: Implications for capsid assembly. J. Virol. 73:2298-2308.

3. Lee, C. (1982): Personal Communication.

4. Miller, M.F., Allen, P.T., and Dmochowski, L. (1973). Quantitative studies of oncornaviruses in thin section. J. Gen. Virol. 21:57-.

5. Miller, M.F. and Rdzok, E.J. (1981). Improved virus particle counting by direct sedimentation onto EM grids. Proc. Annu. Meet., Electron Micros. Soc. Am. 39:404.

6. Rhim, J.S., Smith, K.O., and Melnick, J.L. (1961) Complete an coreless forms fo reovirus (ECHO 10). Ratio f number of virus particles to infective units in the one-step cycle. Virology 15:428-435.

7. Wahl-Jensen, V.M., Kurz, S.K., Hazelton, P.R., Schnittler, H-J., Ströher, U., Burton, D.R., and Feldmann, H. (1974). The role of ebola virus secreted glycoproteins and virus-like particles in activation of human macrophages. Journal of Virology, 79:2413-2419.

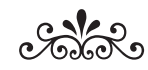

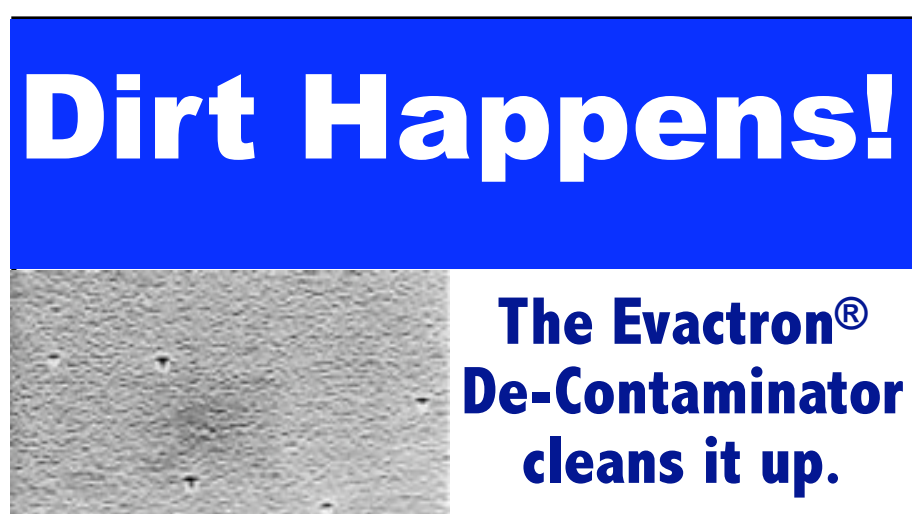

- After first Evactron.

cleaning for 10 minutes

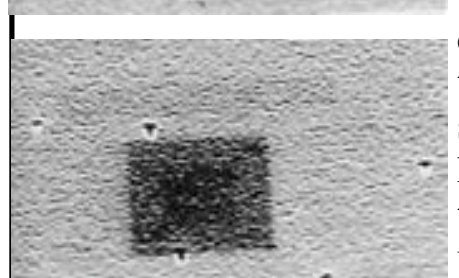

From the most persistent contamination problems to the subtlest, the Evactron system quickly removes the hydrocarbon dirt from Electron Microscopes and vacuum chambers no matter

AMD XL40 Before cleaning what the source.

The needs of nanotechnology have steadily pushed up the performance of FE SEMS in the last decade. We can see smaller details, but ever smaller amounts of dirt can interfere. Dry vacuum systems have become the standard of the industry, but hydrocarbon contamination problems persist because the world we live in provides a constant organic background. Like regular dusting and vacuuming cleans your home, regular use of the Evactron De-Contaminator will remove this dirt and keep it from becoming a problem.

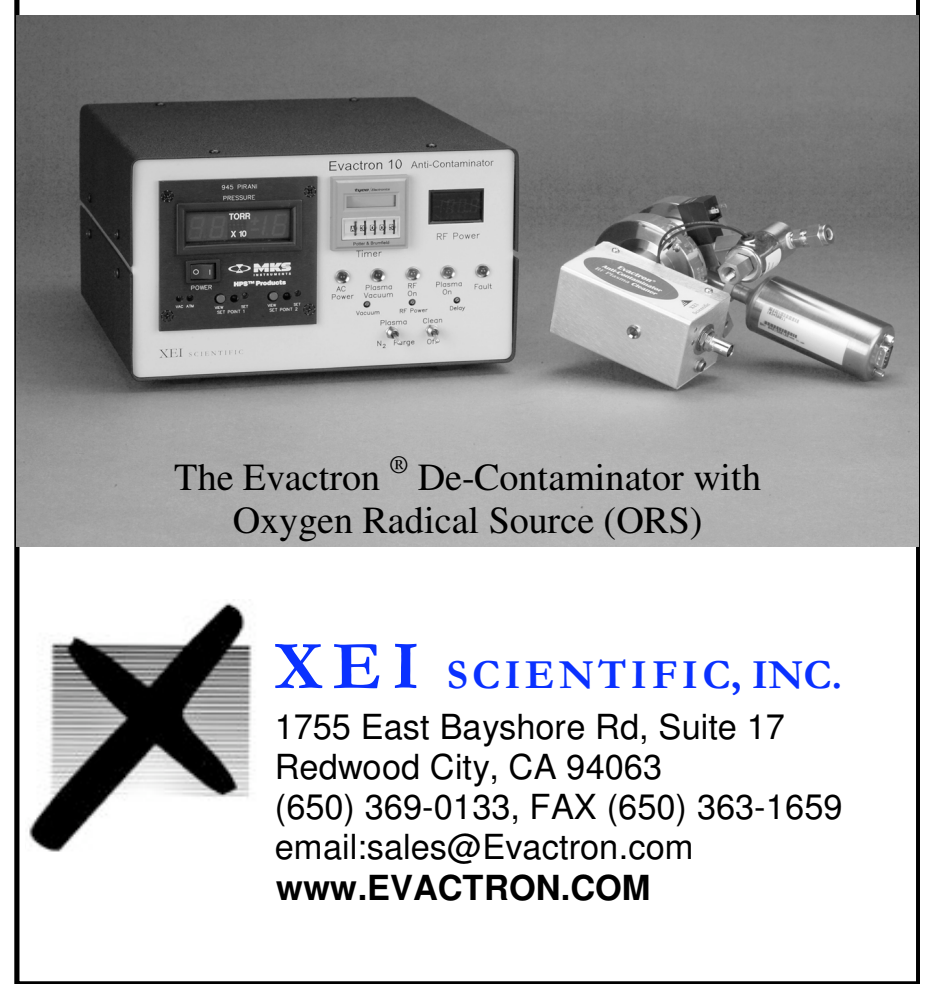

